\title{
DETECTION OF OIL TANK FROM High RESOLUTION REMOTE SENSING IMAGES USING MORPHOLOGICAL AND STATISTICAL TOOLS
}

\author{
D. Chaudhuri ${ }^{1}$ and I. Sharif ${ }^{2}$ \\ ${ }^{1}$ DRDO Integration Centre, Panagarh Base, Muraripur, \\ Burdwan - 731219, W.B., India \\ ${ }^{2}$ Defence Research \& Development Establishment (DRDE), Jhansi Road, \\ Gwalior-474002, Madhya Pradesh, India
}

\begin{abstract}
Oil tank is an important target and automatic detection of the target is an open research issue in satellite based high resolution imagery. This could be used for disaster screening, oil outflow, etc. A new methodology has been proposed for consistent and precise automatic oil tank detection from such panchromatic images. The proposed methodology uses both spatial and spectral properties domain knowledge regarding the character of targets in the sight. Multiple steps are required for detection of the target in the methodology -1) enhancement technique using directional morphology, 2) multi-seed based clustering procedure using internal gray variance $(I G V), 3)$ binarization and thinning operations, 4) circular shape detection by Hough transform, 5) MST based special relational grouping operation and 6) supervised minimum distance classifier for oil tank detection. IKONOS and Quickbird satellite images are used for testing the proposed algorithm. The outcomes show that the projected methodology in this paper is both precise and competent.
\end{abstract}

\section{KEYWORDS}

Recognition, remote sensing, resolution, enhancement, supervised procedure, clustering, minimal spanning tree (MST).

\section{INTRODUCTION}

Extractions of features from satellite based images are significant assignment in various applications. Automatic detection of geographic objects such as bridges [1], buildings [2]-[5], ocean disturbance features [6], roads [7] etc from satellite images is useful in many essential purposes including the construction and preservation of correct physical databases, evaluation of the degree of destruction after natural calamity such as floods or earthquakes and military operations.

Few automatic oil tank detection algorithms are accessible in the literatures [8], [16]. Among them, template matching [9] and Hough transform [10]-[12] are common methodologies. There are some drawbacks in the template matching technique - a) time consuming and b) choice of template. Shape is an important feature for target detection and oil tank is mostly a circular shape feature in the image, which may detect by Hough transform [10], [12]. Due to noise or poor 
illumination Hough transform failed to detect oil tanks very well unless good quality enhancement technique is imposed for isolation the foreground and background.

Multiple steps technique - image fusion, Canny edge detector Hough transform and fast matching method is proposed by Zhang et al. [13]. Wang et al. [14] suggested a very simple method using SAR, which is used for bright spot detection and optical image, which is used for detection of shape feature of all those bright spot. This method [4] is depended on both SAR and visible band images in the same region and this is the major drawback of this method because extra step for registration is required, which is critical for such kind of images. Another technique based on image fusion and morphological operations is suggested by Qiang et al. [15]. Kushwaha et al. [16] proposed a method to extract bright oil tanks by morphological operation. But the method is unable to detect dark oil tanks. Xuan and Yunqing [25] proposed an oil tank detection technique by three steps approach - i) advance visual saliency model are used for separation of oil tanks from the heterogeneous background, ii) detected circular shadow regions and iii) graph search method and prior knowledge are applied for removal of false oil tanks. Recently Zalpour et al. [26] proposed a multi-steps approach for oil tank detection. First they have extracted ROI by using R-CNN and then circles are detected from ROI. Next, features are extracted by using CNN and HOG feature extractors. Lastly, for classification of oil tanks they have used SVM. The method is computationally expensive.

The proposed method is to detect oil tanks from high resolution PAN images using various steps. The basic steps are: 1) directional morphological enhancement module to enhance the required objects, 2) detection and clustering the internal gray variance (IGV), 3) binarization and thinning operation, 4) circular object detection by Hough transform, 5) MST based spatial relationship grouping operation and 6) supervised classification based on statistical and texture features. The algorithm is tested on a variety of images and the results are adequate. Also we have compared our method with Xuan and Yunqing [25] method. It has been observed that two oil tanks with low intensity values of an image are not able to detect by Xuan and Yunqing [25] method. Same oil tanks are detected by the proposed algorithm because the advance directional enhancement technique is able to highlight beautifully both the oil tanks. As a result we are able to isolate these targets from the background by the proposed multi-seed based clustering technique. The remainder of the paper is organized as follows. The proposed oil tank detection methodology is presented in Section 2. Experimental results are discussed in Section 3. We conclude with a summary in Section 4.

\section{Oil TANK Detection Methodology}

The detection of oil tank from high-resolution PAN images is the centre of focus. The research is very useful for defence aspects as well as civilian aspect. Normally segmentation is one of the major steps in the target detection problem. In the proposed technique we are not segmented the target from the background. We are more concentrated in the enhancement technique by which the targets are more prominent than the background. Then we have transformed the enhanced image into feature domain and then we developed a clustering technique of the feature image for isolation possible oil tanks. Finally, three important step properties are used - shape property, neighbouring relational property and supervised classification property. The overall methodology of the proposed algorithm is shown in Figure 1. Each step is described in the next subsequent sections. 


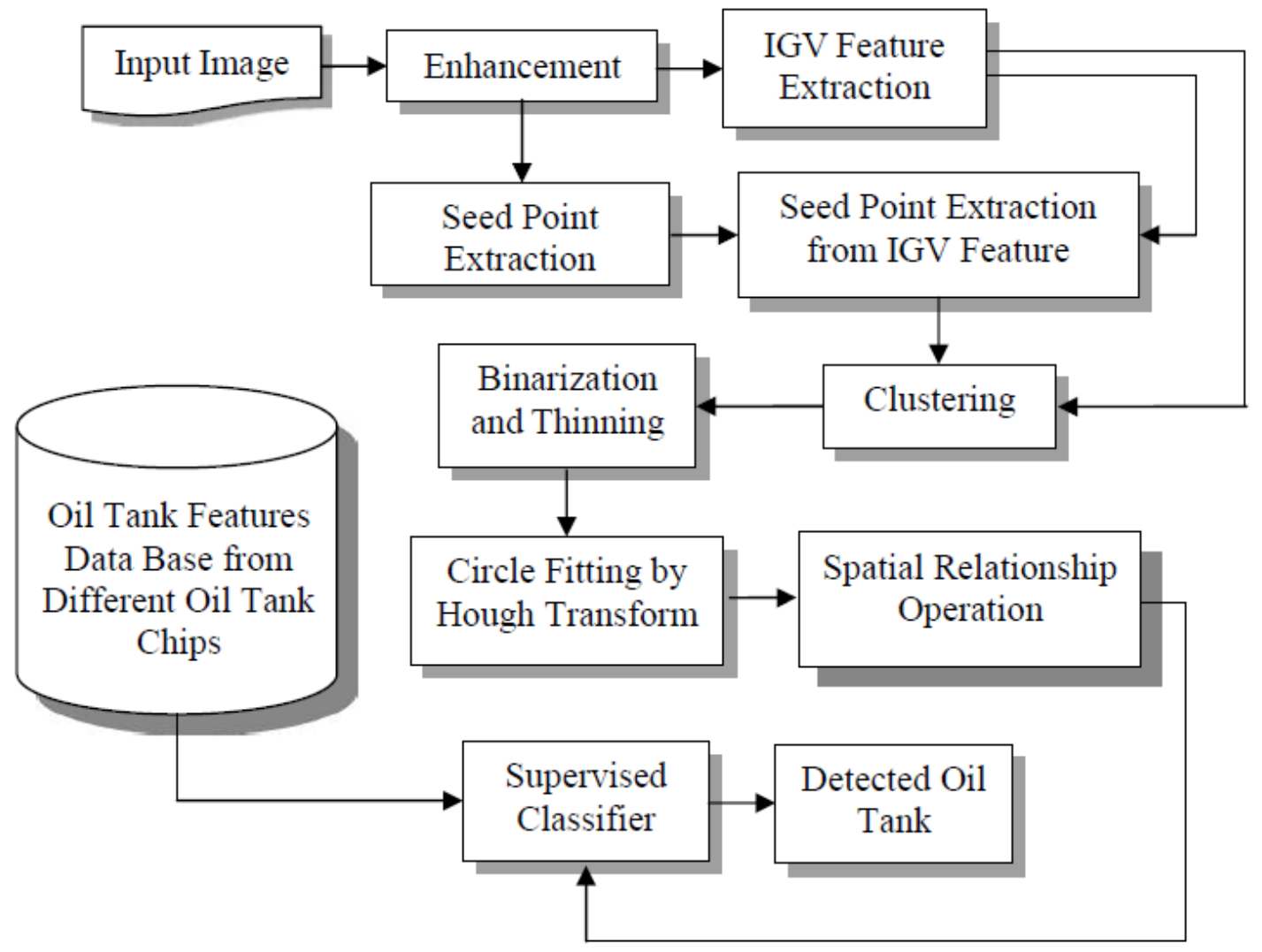

Figure 1. Schematic of the proposed algorithm

\subsection{Image Enhancement}

Better visualization effect and better contrast between the object and non-object in the image are the main aspect of enhancement technique. The purpose of enhancement technique is that object of interest is more homogeneous than non-interested objects. A good enhancement technique will help for isolation of interested object from the background. Morphological filter [17, 18] is an effective tool for such type of operation. A novel directional morphological filtering technique has been proposed by Chaudhuri et al. [7] for enhancement of the object of interest. The same enhancement technique [7] has been applied in the present paper. First select the template of size $5 \times 5$ of the object, which is called the structuring element. We have considered four directions horizontal $\left(0^{0}\right)$, right diagonal $\left(45^{0}\right)$, vertical $\left(90^{\circ}\right)$ and left diagonal $\left(135^{\circ}\right)$ within a $5 \times 5$ window. The proposed enhancement is the directional morphological technique. The choice of direction can be determined by computing standard deviation among the four directions within the $5 \times 5$ window of the original image is minimum. Alternate sequence of opening and closing operations are applied to the original image within the $5 \times 5$ window by using the structuring element along the minimum directional standard deviation. This operation is followed for the whole image and obtained the final enhanced image.

\subsection{IGV Feature Extraction}

The region of interest is more homogeneous than the border part of the region by the previous image enhancement technique. If the region or object in the image is homogeneous then the internal gray variance (IGV) [2] will be very small and consequently in the border region, IGV will be higher than interior body of the object. 
Here, a moving window $W \times W(W=5)$ is considered and compute the mean gray value of the image at the present window position. The candidate pixel is the middle pixel of the window and the value is replaced by the square sum difference from each pixel from mean value of the image at present window position. It has been observed that the deviation at the border region of the manmade object (like, oil tank) is high than the natural object.

\subsection{Seed Point Extraction Technique}

The isolation of manmade and natural objects from a scene of the panchromatic image is very difficult task due to unknown classes of materials, overlie of the brightness values of the classes and multi-modal characteristics of a particular class. As a result, it is very hard job to segment the unprocessed image, particularly synthetic and non-synthetic (natural) classes. To defeat this we have changed the enhanced image into IGV attribute space and then a seed based clustering technique has been applied to separate the artificial object from the rest image.

The basic concept of clustering based on seed point is selection of initial seed point and grow cluster surround the seed. Most beautiful nature of seed based cluster is that it can separate 100\% correct if the data classes are circular and well separated. It is unable to cluster correctly if the data is elongated or complex. Chaudhuri et al. [22], [23] proposed multi-seed concept to overcome this situation.

Present seed point detection technique is based on our previous work [2]. Here, seed points are detected from the domain information of the enhanced image and IGV feature values. First, seed points are extracted using a multi-seed technique of enhanced image, which is described in Algorithm A. Lastly, the final seed points of IGV feature values, variance seeds (VS) [2] are extracted with the help of the seed points of enhanced image as Algorithm B. IGV feature space is clustered by using $V S$, which is described in Section D. The interested reader can go through our previous work [2] and here both the algorithms are described for better understanding.

\section{Algorithm A:}

Step 1: Input image is the enhanced image (current region, $I$ ). Find $g r_{\min }$ and $g r_{\max }$ are the minimum and maximum gray values. Let $g r_{i}$ and corresponding $h r_{i}, i=1,2, \ldots, V$ are the gray value of and frequency, respectively.

Step 2: Compute mode of region $I$ i.e. $m=\max _{j=g r_{\min }}^{g r_{\max }}\left\{h r_{j}\right\}$.

Step 3: Find standard deviation $(S t d)$ w. r. t. mode $(m)$ for region, $I$. That is,

$$
S t d=\left[\frac{1}{\sum_{i=g r_{\max }}^{g r_{\max }} h r_{i}} \sum_{i=g r_{\min }}^{g r_{\max }}\left(m-g r_{i}\right)^{2} h r_{i}\right]^{1 / 2}
$$

Step 4: Select threshold $T_{1}$ (homogeneity factor) and if $S t d>T_{1}$ then follow Step 5. Otherwise follow Step 8.

Step 5: Select the parameter $v$, Gaussian multiplier and accumulate the set of pixels with gray values $g r_{i}$ satisfy the constrains, $m-v \times S t d \leq g r_{i} \leq m+v \times S t d$. 
Step 6: Eliminate all $g r_{i} \in[m-v \times S t d, m+v \times S t d]$ from region $I$. Residual pixel gray values are separated into two sets - i) $g r_{i} \in\left[g r_{\min }, m-v \times S t d\right)$ and ii) $g r_{i} \in\left(m+v \times S t d, g r_{\max }\right]$.

Step 7: If both or any one of the set in Step 6 are non-homogeneous or bigger set then reiterate Steps 1-6.

Step 8: Stop.

Suppose the extracted $k$ seed points by Algorithm A are $m_{1}, m_{2}, \ldots, m_{k}$; which are mode of corresponding $k$ homogeneous clusters, $C_{j}, j=1,2, \ldots k$. Next these seed points and corresponding clusters $\left(m_{j},\left\{C_{j}\right\}\right)$ will be utilized in the IGV feature space (Algorithm B) for extraction $k$ seed points in the feature domain.

\section{Algorithm B:}

- Find the set $P X_{j}=\left\{\left(I V_{x j}, I V_{y j}\right)\right\}$ from IGV feature space for all pair $\left(m_{j},\left\{C_{j}\right\}\right)$ of the image space, for $j=1,2, \ldots, k, x=1,2, \ldots, R$ and $y=1,2, \ldots, S$ where $R$ and $S$ are the rows and columns of the image.

- Extract $I G V$ values for corresponding $\left(I V_{x j}, I V_{y j}\right)$ i.e. $I G V\left(I V_{x j}, I V_{y j}\right), j=1,2, \ldots, k$.

- Compute $G V[j]=\sum_{\forall\left(I V_{x j}, I V_{y j}\right) \in P X_{j}} I G V\left(I V_{x j}, I V_{y j}\right), j=1,2, \ldots, k$

Compute seed in the IGV feature space, $I V S[j]=\frac{G V[j]}{\# P X_{j}}, j=1,2, \ldots, k$ where "\#PX”" is the total number of points of the group $P X_{j}$.

\subsection{Clustering Technique}

The proposed clustering technique is based on multi-seed clustering technique [22], [23]. It has been observed that many small clustered regions are formed after applying the proposed clustering technique to enhanced image and identification oil tank is a hard job from such clustered image. This problem can be handled by nearest neighbor clustering technique in IGV feature space using $V S[j], j=1,2, \ldots, k$. The manmade structures are isolated in a single group by applying this clustering technique. So, the automatic threshold detection is very easy from such clustered image for formation of binary image.

\subsection{Threshold and Thinning Techniques}

The previous IGV feature space cluster image is a gray level image with limited gray variance and it is very easy to convert a binary image for isolation of manmade and natural objects by simple threshold technique, provided appropriate selection of threshold value. There are various automatic threshold value detection techniques in the literature. In this paper, bimodality detection approach [23] is used for detection of automatic threshold value.

The data is said to be bimodal if the data can be divided into two sub-data. Suppose $Q$ be the data population of the IGV cluster image. $Q$ is divided into two parts, say $Q_{L(u)}, Q_{H(u)}$ in such a way so that i) $Q_{L(u)}$ includes all the data with group value $\leq u$, and $Q_{H(u)}$ includes all the data with group value $>u$. and ii) the variances of $Q_{L(u)}$ and $Q_{H(u)}$ are small in respect the variance of $Q$. 
Suppose $N$ and $\sigma^{2}$ are the total data frequency and variance of $Q, N_{L(u)}$ and $\sigma_{L(u)}^{2}$ be the data frequency and variance of $Q_{L(u)}$ and lastly, $N_{H(u)}$ and $\sigma_{H(u)}^{2}$ be the data frequency and variance of $Q_{H(u)}$. Now for estimation the threshold value, say $u=T$ (bimodality parameter), we will construct the objective function, which will be minimum among all the gray values of IGV cluster image as

$$
O B(u)=\frac{N_{L(u)} \sigma_{L(u)}^{2}+N_{H(u)} \sigma_{H(u)}^{2}}{N \sigma^{2}}
$$

So $T$ will be the threshold value for thresholding IGV cluster image, $\left(I G V_{C l}\right)$ to binary image as

$$
I G V_{\text {Binary }}(x, y)=\left\{\begin{array}{l}
0 \text { if } I G V_{C l}(x, y)<T \\
1 \text { if } I G V_{C l}(x, y) \geq T
\end{array}\right.
$$

Oil tank is the circular shape object and the edge information is very important for finding the shape. Though $I G V_{\text {Binary }}$ image gives the edges of the manmade object but the edges are thicker and thinning algorithm, which is available in the literature [24] is applied for finding the proper edge.

\subsection{Circle fitting}

Feature extraction in images is one of the most challenging tasks in computer vision. Practically, objects of interest may appear in different shapes and sizes and a solution of this problem is to find an algorithm for extraction any shape and size within an image. Then the objects can be classified accordingly to parameters needed to describe the shapes and most effective method for this is the Hough Transform [12], [19]. In this paper we have extracted circular shapes objects from the binary image by using Hough transform.

\subsection{MST Based Spatial Relationship Grouping Operation}

Oil tank is an important defence target and normally they formed in a group i.e. many oil tanks are constructed in an open space connected with roads. Isolate tank is not so much important because that may be water tank or other object. In this paper, we proposed Minimal Spanning Tree (MST) based clustering technique for formation cluster of oil tanks. The idea is very simple. Suppose $K$ number of circular shape objects, $O C_{i}, i=1,2, \ldots, K$ are detected by the previous steps. First compute the centroids of all the circular shape objects, which are the nodes of the graph and construct the MST between all the centriods. The edge weights between the two connected nodes are computed by the Euclidean distance between two connecting nodes. These edge weights will make the decision that whether the connected nodes will form a group or not. Suppose $T_{G p}$ is the distance threshold value, which depends on the resolution of the image and the decision logic is if the edge weight between the two connected objects is greater than $T_{G p}$ then those objects are formed different clusters; otherwise they are in the same cluster. Experimentally we have seen $T_{G p}=12$ gives the good result for IKONOS and QuickBird data.

\subsection{Supervised classification}

The possible oil tanks along with other similar objects are detected from the previous subsections. A supervised classifier has to be develop for detection of confirm oil tanks. Image target chips of oil tanks are stored as training samples and 15 different statistical and texture features [20] are 
extracted from those training sample oil tank chips. So, each oil tank chip is having a 15 dimensional feature vector space, $O_{F}$. Now for the classification, we have extracted the same 15 dimensional features from each possible oil tanks of the output image of the previous step and compute the distances between the 15 dimensional feature vector of the possible oil tank and the training data base $O_{F}$. Find the minimum distance among all distances and if the minimum distance is less than the threshold value, $T_{C}$ then the object is a confirm oil tank. At the same time, we have seen the minimum distance between the similar object feature vector, which is not an oil tank and training data base vector $O_{F}$ is greater than the threshold value, $T_{C}$. In our case, $T_{C}=0.4$ is a good choice for IKONOS and QuickBird data.

\section{EXPERIMENTAL RESULTS}

We have tested the various steps of the proposed algorithm in panchromatic images of IKONOS and QucickBird satellites. All the steps of the algorithm are executed and timed on an HP xw6400 workstation (Intel(R) Xeon(R), 5130 at $200 \mathrm{GHz}, 2.00 \mathrm{~GB}$ RAM, Microsoft windows $\mathrm{XP}$ ). Total computational cost of oil tank detection by the proposed algorithm is 312 milliseconds.

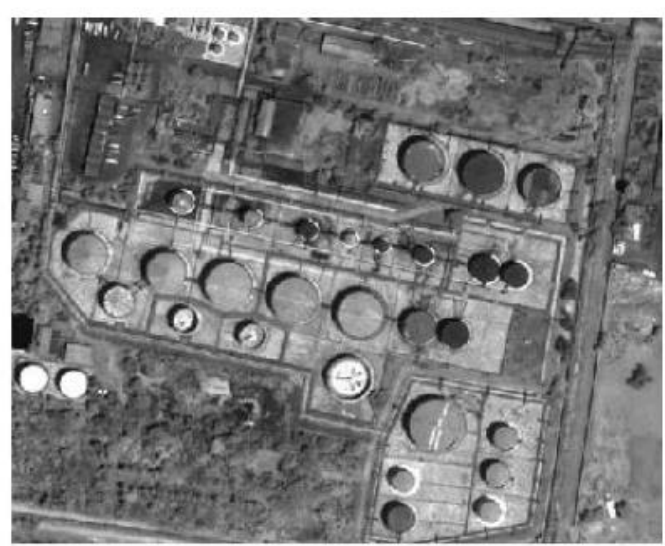

(a)

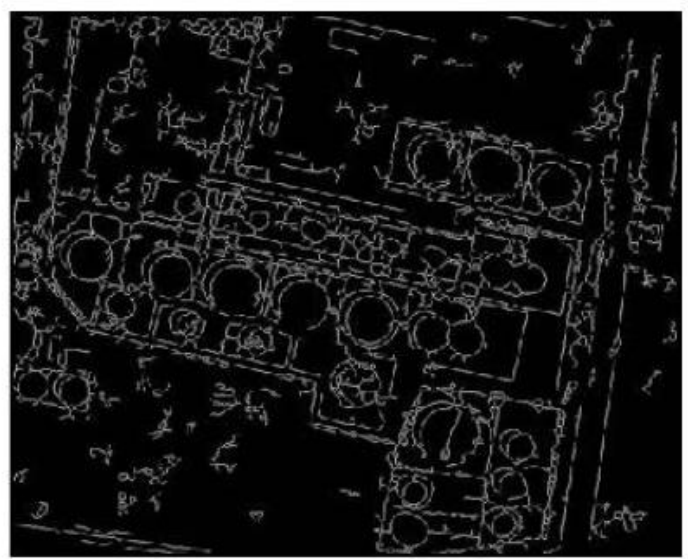

(c)

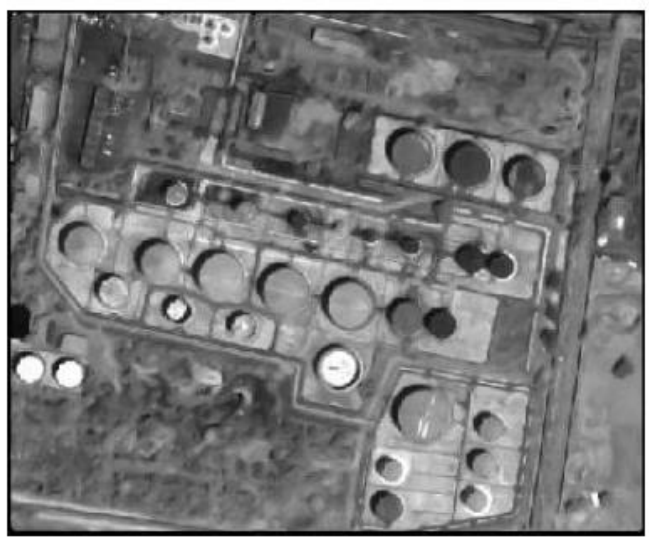

(b)

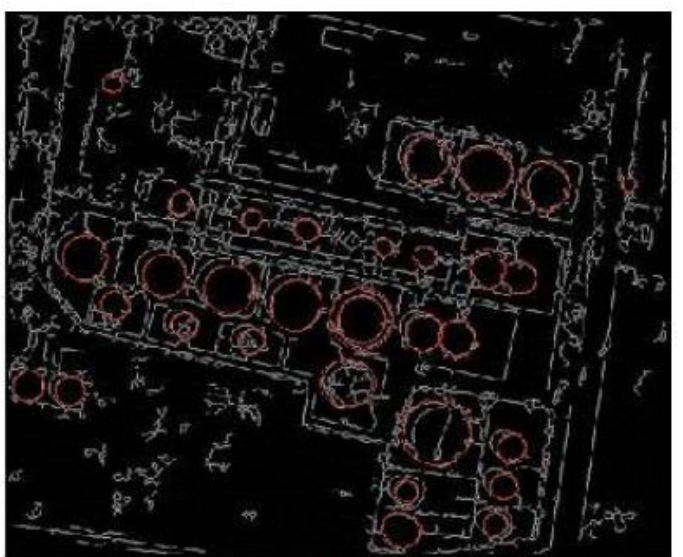

(d) 


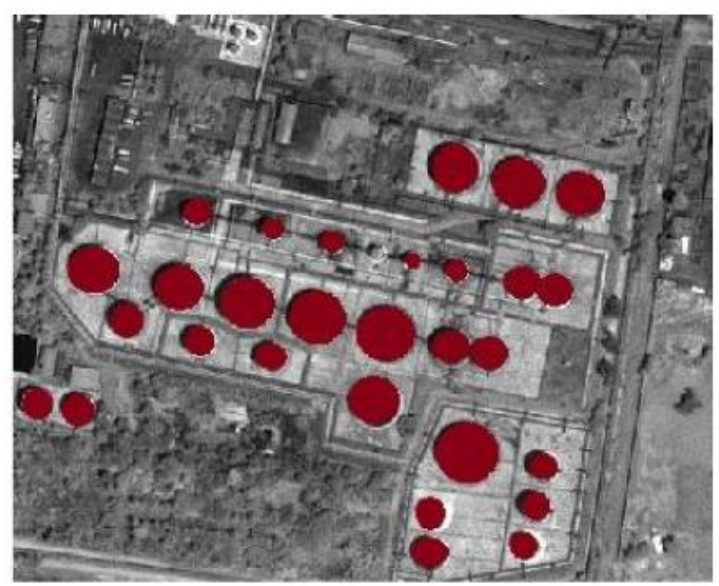

(e)

Figure 2. IKONOS Panchromatic image: (a) original image, (b) enhanced image, (c) binary image, (d) circular shape detected image, and (e) final output

The original IKONOS satellite image of size $493 \times 401$ is shown in Figure 2 (a). The proposed enhancement technique has been applied to the original image and Figure 2(b) shows the enhanced image. It is noticed that manmade structures in Figure 2(b) are more uniform and enhanced than the original structures in Figure 2(a). Figure 2(c) shows the segmented image by using the proposed clustering technique of the IGV feature space. The detected circular shape objects by using Hough transform is shown in Figure 2(d). Few flash alarms are appeared in Figure 2(d) and removing those unwanted objects final output is shown in Figure 2(e).

Figure 3(a) and Figure 4(a) show the PAN images from different satellites IKONOS and QuickBird of sizes $910 \times 610$ and $78 \times 109$, respectively. The detected oil tanks are shown Figure 3(b) and Figure 4(b), respectively by the proposed algorithm.

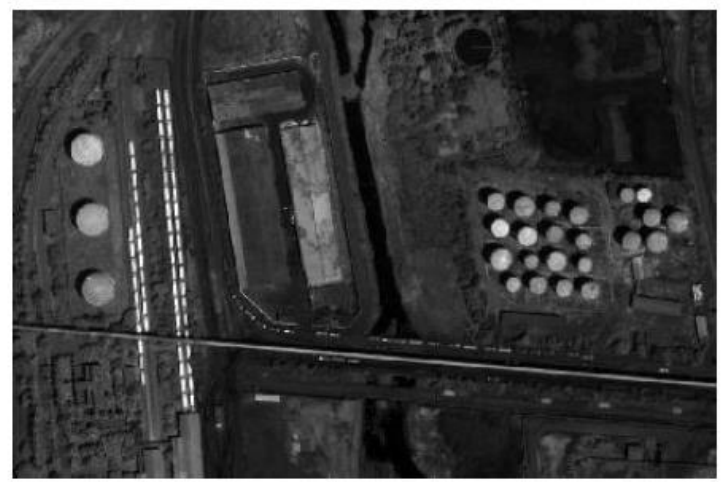

(a)

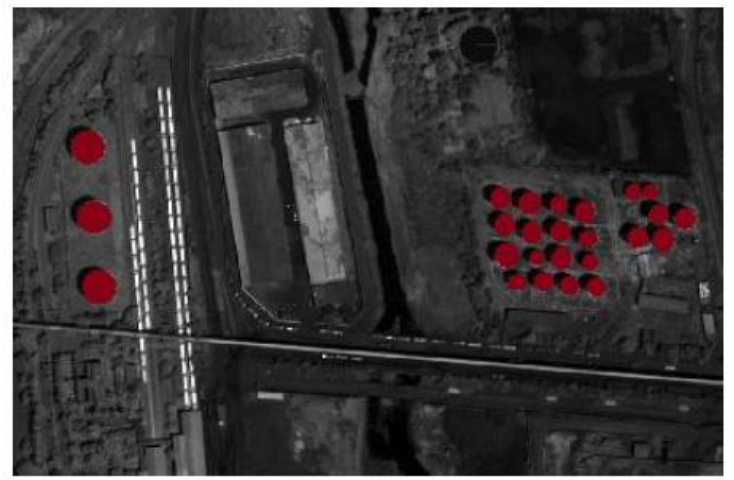

(b)

Figure 3. IKONOS PAN image: a) original image and (b) output image 


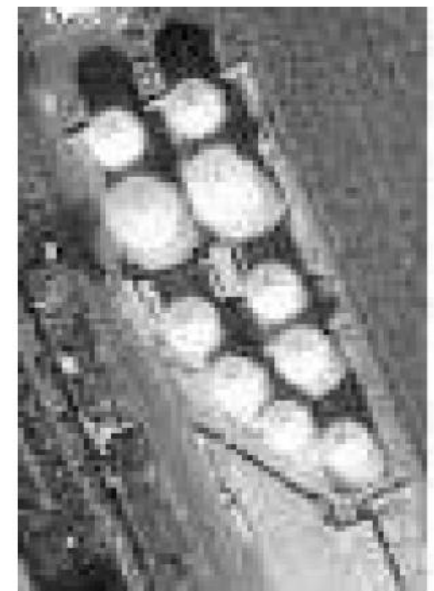

(a)

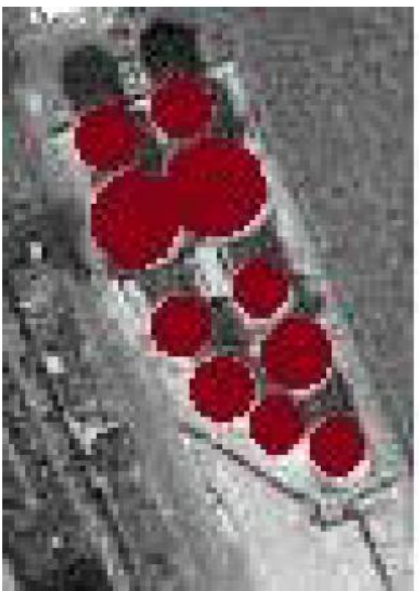

(b)

Figure 4. QuickBird PAN image: (a) original image and (b) output image

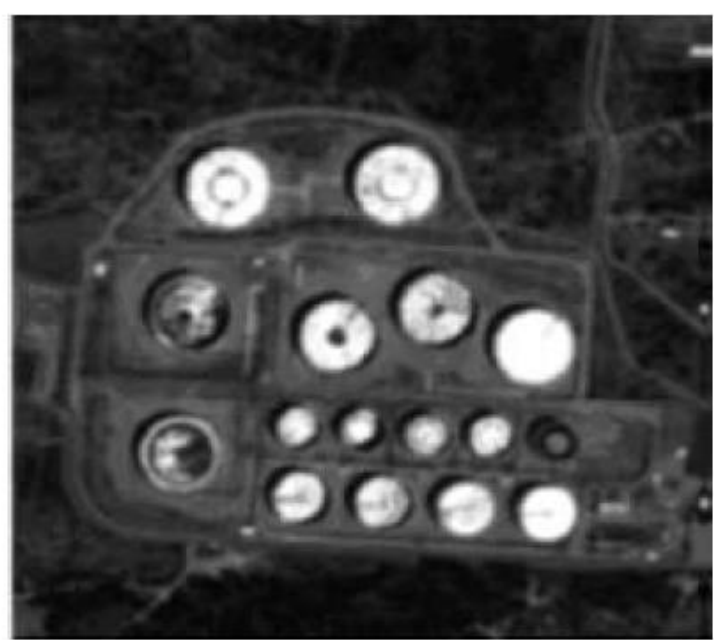

(a)

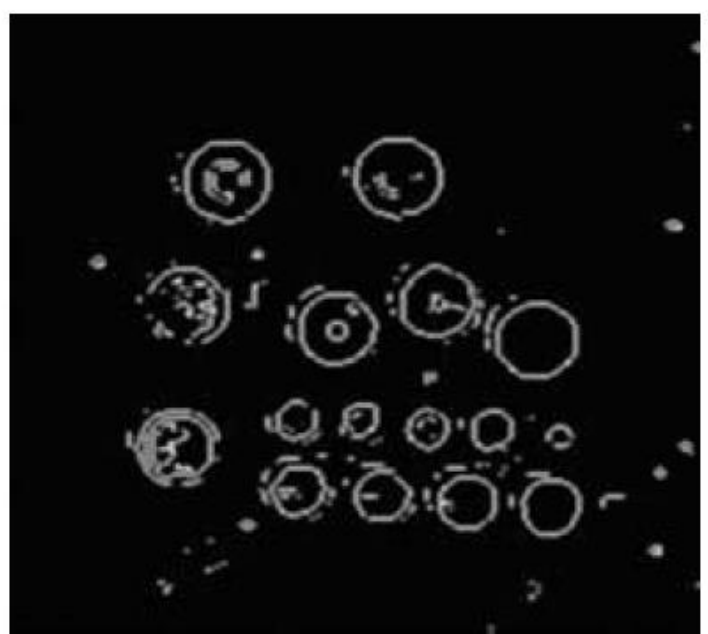

(b)

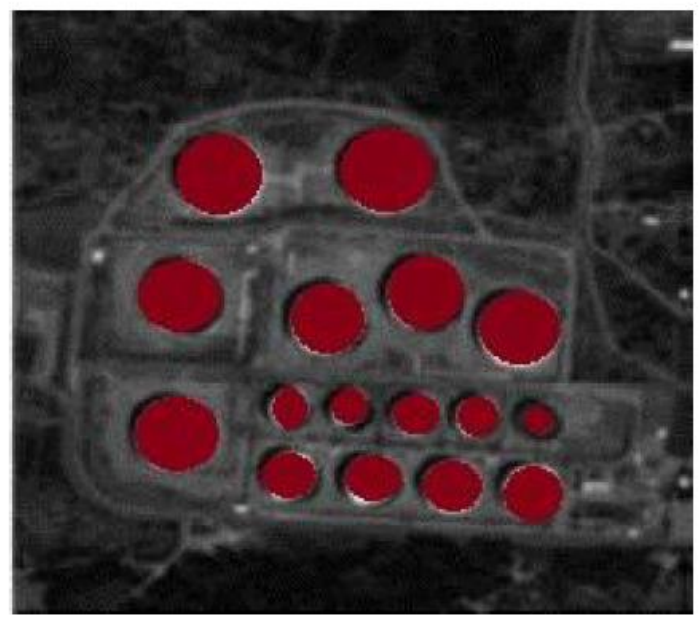

(c)

Figure 5: Original IKONOS image: (a) Original image of size $254 \times 225$, (b) detected oil tanks by Xuan and Yunqing [25] and (c) detected oil tanks by the proposed method 


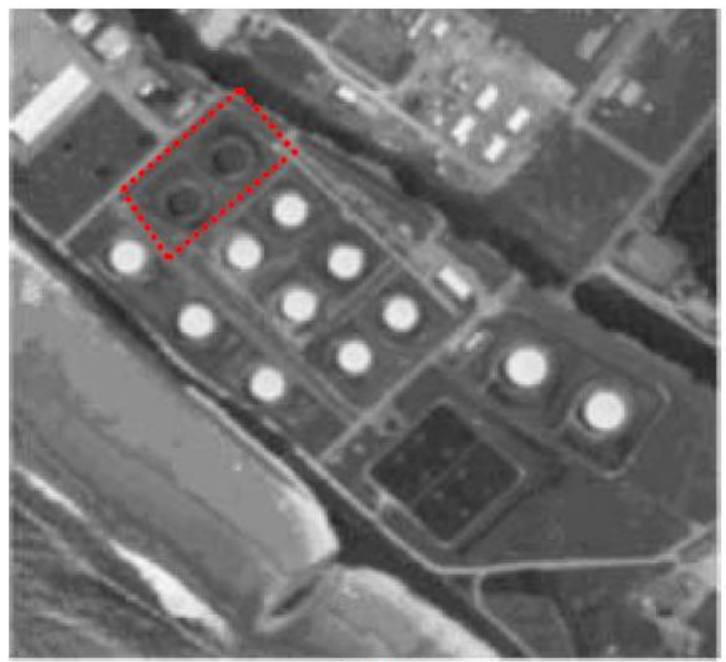

(a)

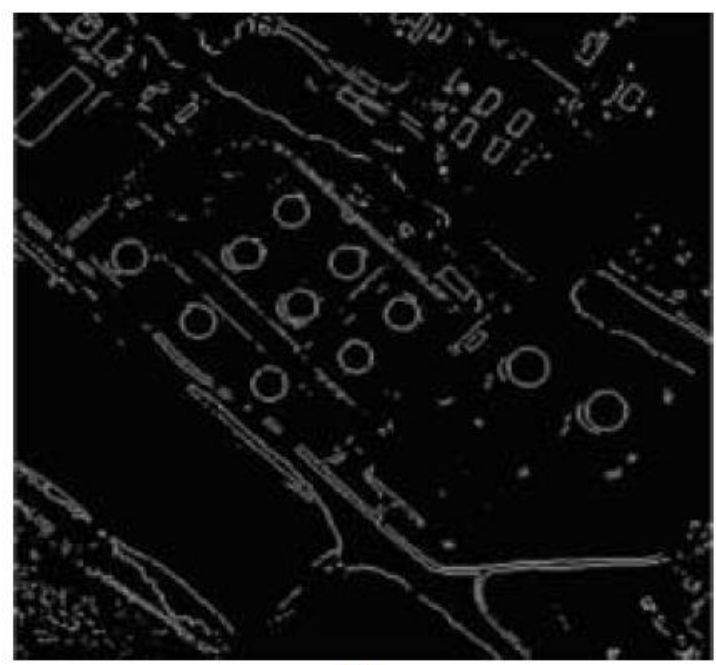

(b)

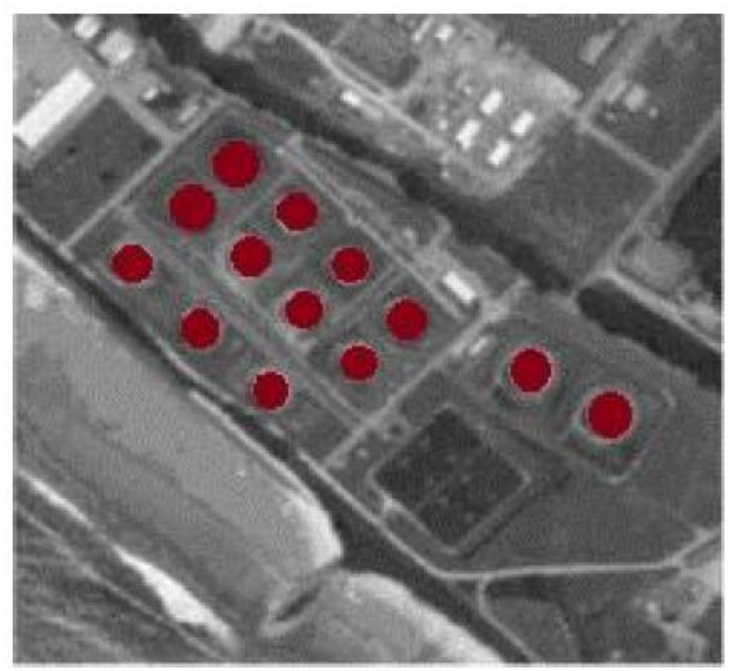

(c)

Figure 6: Original IKONOS image: (a) Original image of size 246×226, (b) detected oil tanks by Xuan and Yunqing [25] and (c) detected oil tanks by the proposed method

Figure 5(a) shows an original IKONOS image of size $254 \times 225$. The detected oil tanks by Xuan and Yunqing [25] and the proposed method are shown in Figures 5(b) and 5(c), respectively. We have seen that both the methods are detected oil tanks correctly.

Another IKONOS image is shown in Figure 6(a) of size $246 \times 226$. The detected oil tanks by Xuan and Yunqing [25] and proposed method are shown in Figures 6(b) and 6(c), respectively. Here we have seen that two black oil tanks inside the dotted red color rectangular shape in the original image Figure 6(a) are not detected by Xuan and Yunqing [25] method. At the same time, both the oil tanks are detected by the proposed technique. It is because of enhancement technique which is highlighted both the oil tanks perfectly and subsequent clustering technique of the proposed method is able to isolate these targets from the background. 


\section{Conclusions And Future Scope}

Multi-steps algorithm have described in this paper for automatic extraction of oil tank from high resolution PAN satellite images. This problem has many applications for civilian, commercial, and military domains. The significant modules in the projected algorithm are: image enhancement for highlight the target from the background, multi-seed based clustering technique using internal gray variance, binarization and thinning operation, circular object detection using Hough transform, MST based spatial relationship cluster formation and supervised classification using statistical and texture features. A huge amount of training images of the various oil tanks have been generated and 15 dimensional statistical and texture features have extracted for supervised classification module. The proposed algorithm was tested on IKONOS and QuickBird satellites PAN images and satisfactory results have reported by the proposed algorithm. Also we have compared our results with other method and it has observed that the proposed algorithm gives the better results.

Presently machine learning is an advance method for target detection in supervised manner. Different high resolution sensor data are available. Our future work includes the development of algorithms to identify oil tanks from panchromatic high-resolution satellite imagery by using machine learning.

\section{REFERENCES}

[1] Chaudhuri, D. \& Samal A., (2008) "An automatic bridge detection technique for multi-spectral images," IEEE Trans. on Geoscience and Remote Sensing, Vol. 46, No. 9, pp. 2720-2727.

[2] Chaudhuri, D., Kushwaha, N. K., Samal, A. \& Agarwal, R. C., (2016) "Automatic building detection from high-resolution satellite images based on morphology and internal gray variance", IEEE Journal of Selected Topics in Applied Earth Observations and Remote Sensing, Vol. 9, No. 5, pp. 1767-1779.

[3] Theng, L. B., (2006) "Automatic building extraction from satellite imagery", J. Engineering Letters, Vol. 13, No. 3, EL_13_3_5 (Advance online publication), 4 November.

[4] Xiaoying, J. \& Davis, C. H., (2005) "Automated building extraction from high-resolution satellite imagery in urban areas using structural, contextual and spectral information", Journal of Applied Signal Processing, Vol. 14, pp. 2196 - 2206.

[5] Khoshelham, K., Nardinocchi, C., Frontoni, E., Mancini, A. \& Zingaretti, P., (2010) "Performance evaluation of automated approaches to building detection in multi-source aerial data", ISPRS Journal of Photogrammetry and Remote Sensing, Vol. 65, pp. 123 - 133.

[6] Chaudhuri, D., Samal, A., Agrawal, A., Sanjay, Mishra, A., Gohri, V., \& Agarwal, R. C., (2012) “A statistical approach for automatic detection of ocean disturbance features from SAR images", IEEE Journal of selected topics in Applied Earth Observations and Remote Sensing, Vol. 5. No. 4, pp. 1231 $-1242$.

[7] Chaudhuri, D., Kushwaha, N. K., \& Samal, A., (2012) "Semi-automated road detection from high resolution satellite images by directional morphological enhancement and segmentation technique", IEEE Journal of selected topics in Applied Earth Observations and Remote Sensing, Vol. 5, No. 5, pp. $1538-1544$.

[8] Zhu, C., Liu, B., Zhou, Y., Yu, Q., Liu, X., \& Yu, W., (2012) "Framework design and implementation for oil tank detection in optical satellite imagery", IEEE International Geoscience and Remote Sensing Symposium, THP.P11, July, Munich, Germany, pp. $22-27$.

[9] Gonzalez, R. C. \& Woods, R. E., (2008) Digital Image Processing, Pearson Education, Delhi, India.

[10] Illingworth, J. \& Kittler, J., (1988) "A survey of the Hough transform computing", Computer Vision, Graphics and Image Processing, Vol. 44, No. 1, pp. $87-116$.

[11] Wei, Y., (1998) "Improved dynamic broad sense Hough transform and its application on round detection", Surveying and Mapping Information and Engineer, Vol. 4, pp. 23 - 26.

[12] Wu, M., Song, Z., Li, B., Li, F., Li, B., \& Shen, C., (2015) "A method to detect circle based on Hough transform", Int. Conf. on Information, Machinery, Materials and Energy (ICISMME), Atlantis Press, pp. $2028-2031$. 
[13] Zhang, W., Zhang, H., Wang, C., \& Wu, T., (2008) "Automatic oil tank detection algorithm based on remote sensing image fusion", IEEE International Geoscience and Remote Sensing Symposium (IGARSS), Vol. 6, pp. $3956-3958$.

[14] Wang, Y., Tang, M., Tan, T., \& Tai, X., (2004) "Detection of circular oil tanks based on the fusion of SAR and optical images", Third International Conference on Image and Graphics (ICIG), pp. $524-$ 527.

[15] Qiang, Z., Du, X., \& Sun, L., (2011) "Remote sensing image fusion for dim target detection", International Conference on Advanced Machatronic Systems (ICA Mechs), pp. 379 - 383.

[16] Kushwaha, N. K., Chaudhuri, D. \& Singh, M. P., (2013) "Automatic bright oil circular type oil tank detection using remote sensing images”, Defence Science Journal, Vol. 63, No. 3, pp. 298 - 304.

[17] Haralick, R. M., Sternberg, S. R., \& Zhuang, X., (1987) "Image analysis using mathematical morphology”, IEEE Trans. on Pattern Analysis and Machine Intelligence, Vol. 9, pp. 532 - 550.

[18] Serra, J., (1982) Image Analysis and Mathematical Morphology, New York: Academic.

[19] Duda, R. \& Hart, P. E., (1972) "Use of the Hough transform to detect lines and curves in pictures", ACM, Vol. 15, No. 11, pp. $11-15$.

[20] Haralick, R. M., Shanmugam, K., \& Dinstein, I., (1973) “Texture features for image classification”, IEEE Trans. on Systems, Man and Cybernetics, Vol. 3, pp. 610 - 621.

[21] Cormen, T. H., Leiserson, L. E., Rivest, R. L., \& Stelin, C., (2002) Introduction to algorithm, Prentice Hall of India Pvt. Ltd., Eastern Economy Edition, New Delhi, pp. 570 - 573.

[22] Chaudhuri, D. \& Chaudhuri, B. B., (1997) "A novel multi-seed non-hierarchical data clustering technique," IEEE Trans. on System, Man and Cybernetics, Part - B, Vol. 27, No. 5, pp. 871-877.

[23] Chaudhuri, D. \& Agrawal, A., (2010) "Split-and-merge procedure for image segmentation using bimodality detection approach”, Defence Science Journal, Vol. 60, No. 3, pp. 290-301.

[24] Rosenfeld, A. \& Kak, A., (1982) Digital Picture Processing, New York, Academic.

[25] Xuan, L. \& Yunqing, L., (2016) "Oil Tank Detection in Optical Remote Sensing Imagery Based on Quasi-circular Shadow”, Journal of Electronics and Information Technology, Vol. 38, No. 6, pp. 1489-1495.

[26] Zalpour, M., Akbarizadeh, G. \& Alaei-Sheini, N., (2020) "A new approach for oil tank detection using deep learning features with control false alarm rate in high-resolution satellite imagery", International Journal of Remote Sensing, Vol. 41, No. 6, pp. 2239-2262

\section{AUTHORS}

Dr. Debasis Chaudhuri received the B. Sc. (with Honors) in Mathematics from VisvaBharati University, Santiniketan, the M. Sc. in Applied Mathematics from Jadavpur University, Kolkata, and $\mathrm{Ph}$. D. degree in image processing and pattern recognition from Indian Statistical Institute, Kolkata. He is currently a senior scientist and DGM at DRDO, Panagarh, India. He was a research associate of Council of Scientific and Industrial Research (CSIR) at Indian Statistical Institute, Kolkata. Dr. Chaudhuri was a project scientist at Indian Statistical Institute in ISI-ADRIN Project (collaborated by ISI and Dept. of Space, Govt. of India). He was a Scientist at Defence Electronics

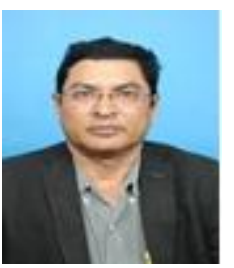
Applications Laboratory, Dehradun, India. He was an associate professor of Defence Institute of Advance Technology (DIAT), Pune. He was a visiting professor at University of Nebraska, USA in the Dept. of Computer Science and Eng. for 2003-2004. He has also visited many other Universities and Institutes within and outside India for delivering invited lectures. He has been a member of the program/organizing committees of many national and international conferences. He has more than thirty years experience in the field of image processing, pattern recognition, computer vision and remote sensing with extensive systems development and implementation experience. He has got lot of experience in advising and guiding others in related fields. He has extensive experience in the field of automatic target detection from satellite imagery. He had successfully completed six national important projects for the services. He had guided many Ph. D/M. Tech/M.S Student/B. Tech students. Two students were awarded the best project award from IEEE at Western Region Software Park, Pune and National Institute of Technology, Nagpur. He has authored or coauthored over 60 papers in international journals and conferences in the area of image processing and pattern recognition. He is reviewer in many international journals. Also he is an Associate Editor of several international journals. He is a senior member of IEEE and fellow of IETE. He has received Technology Award from DRDO Science Forum, Mins. of Defence, Govt. of India in 2011 for excellent contribution in “Target Detection from Remote Sensing Satellite Data”. He has received "Group Technology Award - 
2013" from DEAL, DRDO, Mins. of Defence, Govt. of India for contribution to success the "Image Intelligence Environment" project. He has received Technology Award from DRDO Science Forum, Mins. of Defence, Govt. of India in 2018 for excellent contribution in "Supervised classification of multispectral images by minimum distance with majority must be granted logic using multi-seed technique". His research interests include image processing, pattern recognition, computer vision, remote sensing and target detection from satellite, SAR, Thermal and MMW imageries.

Dr. Imran Sharif received his B. Tech (Computer Science and Engineering) from Institute of Engineering and Technology, Kanpur in 2002 and M. Tech from CDAC, Noida in 2005 and $\mathrm{Ph}$. D. (Computer Science \& Engineering) from Uttarakhand Technical University, Dehradun in 2018. Currently he is working as scientist at DRDE, Gwalior. More than 15 years research experience in the field of Hyper spectral image processing, Pattern recognition, Computer vision, Remote sensing and target detection from satellite imagery and Embedded system. He is a life time member of computer society of India and Indian Society of Remote Sensing.

(C) 2021 By AIRCC Publishing Corporation. This article is published under the Creative Commons Attribution (CC BY) license. 